\title{
Spannungsverhältnisse in Nichtregierungsorganisationen
}

\section{Theresia Wintergerst}

Das Wort Nichtregierungsorganisation oder Non-governmental-Organization (NGO) ist ein Negativbegriff. Es bezeichnet daher, was eine Organisation nicht sein will, gibt aber noch nicht den positiven Gehalt des Begriffs vor. Dies ist ein erster Hinweis darauf, dass NGOs gar nicht so einfach zu fassen sind. Schon wenn man die verschiedenen gesellschaftlichen Sektoren wie Staat und Markt betrachtet, ergeben sich Definitionsfragen. Im staatlichen Bereich üben Nichtregierungsorganisationen politischen Einfluss aus, streben aber nicht die Übernahme von Regierungsmacht an. Was den Markt betrifft, so sind sie in ihrer Zielsetzung oftmals nicht gewinnorientiert, brauchen aber in der Regel for-profitAktivitäten, um ihren Bestand nachhaltig sichern zu können. Der zivile Sektor, dem man die NGOs ohne zögern zuordnen kann, ist allerdings nicht identisch mit den NGOs, zu breit sind die Organisationsformen und -typen, die sich im zivilen Sektor finden. Sicher ist nicht jeder Verein und jede Initiative eine NGO. Grenzen sind zu ziehen, doch diese Grenzen sind eher als „Hecken“ zu verstehen, denn als Mauern. Denn in vielen Bereichen gibt es Übergänge und Überschneidungen.

Der Einführungsartikel führt an ein Verständnis von NGOs heran und begründet dieses. Dabei werden die Wirkungen nach außen und die Wirkungen nach innen erörtert, die man NGOs zuschreiben kann. Man kann dabei die Funktionsweise von NGOs nicht verstehen, ohne sie als Mehrebenengeschehen zu erfassen. Der Einführungsartikel skizziert die Ebenen der NGOs und unterstreicht die Bedeutung der lokalen Ebene. Deutlich wird, dass NGOs durch vielfältige Spannungsverhältnisse gekennzeichnet sind. Armin Stickler weist nach, dass die Zahl der NGOs bereits seit den 1960-er Jahren stieg, und die globale Wertschätzung der NGOs in den 1990-er Jahren einen erheblichen Aufschwung erfuhr. Vor allem auf großen UN-Konferenzen gewannen NGOs an Einfluss (Stickler 2005, S. 54 ff.). Nicht zuletzt die in den 1990-er Jahren gestarteten Agenda 21-Prozesse tragen dieser gestiegenen Bedeutung Rechnung. Darin werden die Regierungen angehalten, governance-Konzepte zu entwickeln, in denen den Akteuren der Zivilgesellschaft ein

\footnotetext{
Online publiziert: 31.08 .2010

(C) VS Verlag für Sozialwissenschaften 2010

T. Wintergerst $(\bowtie)$

Herrenbergweg 12, 86567 Hilgertshausen, Deutschland

E-Mail: maria-theresia.wintergerst@unibw.de
} 
fester Platz in politischen Planungs-, Implementierungs- und Durchführungsprozessen eingeräumt wird. NGOs erheben dabei in verschiedenen Politikfeldern ihre Stimme.

- Ein wichtiger Akteur im Bereich Umwelt ist beispielsweise der Bund für Umwelt-und Naturschutz, der bundesweit rund 400.000 Mitglieder hat.

- Im Bereich der Menschenrechte kann man amnesty international als global player bezeichnen. 2,2 Mio. engagieren sich weltweit für die Verbesserung der Menschenrechtssituation in vielen Ländern der Welt.

Amnesty international beobachtet, wie viele anderen NGOs, ob Regierungen die internationalen Vereinbarungen, die sie unterzeichnet haben, auch einhalten. Tun sie es nicht, skandalisieren sie dies. „It's hard to be selfish in public“, nach diesem Motto stellen viele NGOs Öffentlichkeit her, und arbeiten auf verschiedene Weise an der Verbesserung von Sozial- und Umweltstandards. Weitere Politikfelder, in denen NGOs vertreten sind, sind die Bereiche Frieden, Frauen, Weltordnung und Entwicklung/Empowerment (Stickler 2005, S. 55).

Dieses Themenheft stellt NGOs jedoch nicht nach Themengebieten vor. Dies würde notgedrungen Auslassungen mit sich bringen, die nicht vertretbar wären. Deshalb wählt das hier vorgelegte Themenheft einen anderen Weg. Nach dem einführenden Artikel greift Julia Franz das Thema des intergenerationellen Lernens in NGOs auf. Dies ist für alle NGOs im Binnenraum ein grundlegendes Thema. Das Thema der Generationen verweist auf „Gründungsgeschichten“ von NGOs und deren weitere Entwicklung. Will die NGO über ihre Gründungsgeneration hinaus Bestand haben, muss sie immer wieder Prozesse intergenerationellen Dialogs und Lernen zwischen Menschen verschiedener Lebensalter ermöglichen. In NGOs treffen Menschen aus unterschiedlichen Alterskohorten aufeinander, die durch ihre gesellschaftlich-historische Erfahrung unterschiedlich geprägt wurden. Dies hat großen Einfluss auf deren Engagement und die damit verbundenen Motive. Daraus ergeben sich Herausforderungen des intergenerationellen Lernens, auf die jede NGO eine Antwort finden muss, will sie nachhaltig sein. Julia Franz als Expertin für intergenerationelles Lernen zeigt hier die Spannungsfelder auf.

Im zweiten Artikel wird der Schwerpunkt dieses Themenheftes deutlich. Exemplarisch werden NGOs aus dem Bereich Entwicklungspolitik und Entwicklungszusammenarbeit beleuchtet. Die Fokusierung auf NGOs aus einem Themenfeld erlaubt die multiperspektivische Erfassung von Spannungsverhältnissen, die diesen NGOs inhärent sind.

Norbert Frieters-Reermann führt uns in die Spannungsfelder der NGOs in der Entwicklungspolitik ein. Nur einige sollen hier genannt werden. Entwicklungspolitische NGOs müssen ihren Weg finden zwischen kurzfristiger Nothilfe und langfristiger Entwicklungsarbeit, zwischen Projekten auf der Mikroebene und Strategien für die Makroebene, zwischen Inlandsarbeit und Projektarbeit. Dabei sind NGOs auf die Übereinstimmung von Organisationszielen und Individualzielen in besonderem Maße angewiesen, Glaubwürdigkeit ist ja die zentrale „Währung“ der NGOs, sie brauchen also Mitarbeiter, die sich mit den Organisationszielen voll identifizieren. Ist aber von dieser Übereinstimmung selbstverständlich auszugehen? Norbert Frieters-Reerman, selber langjähriger Mitarbeiter einer entwicklungspolitischen Hilfsorganisation, fragt kritisch: „,Bei entwicklungspolitischen Nichtregierungsorganisationen wäre allerdings zu fragen, welch vielfältigen Konflikte zwischen Organisationszielen und Individualzielen bei genauerem Hinsehen 
deutlich werden und wie mögliche Spannungen und Widersprüche zwischen diesen Zielen in verschiedener Weise zum Tragen kommen. “Diese Frage führt unmittelbar zu der Frage nach den internen Strukturen und Hierarchien, in denen haupt- und ehrenamtliche Mitarbeiter und Mitarbeiterinnen arbeiten und wie diese mit den Organisationszielen übereinstimmen oder eben nicht. Prägnant skizziert Frieters-Reermann ein ganzes Bündel von Spannungsfeldern. Es wird deutlich, dass NGOs eine gewisse Meisterschaft darin brauchen, mit internen und externen Spannungsverhältnissen umgehen zu können.

Die folgenden zwei Artikel machen es konkret. Andrea Mewaldt führt als langjährige selbständige Beraterin der Zivilgesellschaft in postsozialistischen Transformationsländern aus, wie wichtig Lokalisierungsprozesse für NGOs sind. NGOs in der Entwicklungszusammenarbeit dürfen in ihren Wirkungsländern nicht einfach westliche Muster kopieren. Die Qualität der Zusammenarbeit zwischen Geber- und Empfänger NGOs ist besonders wichtig. Wenn Lokalisierungsprozesse nicht gelingen hat dies fatale Folgen: ,, Zieht die Karawane der ausländischen Helfer weiter, geht die notwendige Unterstützung teilweise wieder verloren und muss lokal neu initiiert und mit entsprechenden Strukturen aufgebaut werden. "

Andrea Mewaldt schließt an diese Beobachtungen ihre Kritik an der Förderpolitik an, die kleinere NGOs benachteiligt, obwohl sie einen größeren Lokalbezug aufweisen können. Sie erläutert die negativen Wirkungen von finanzieller Förderung, die sich ausschließlich auf Projekte bezieht und den Erhalt von Strukturen nicht berücksichtigt. Dabei wird ersichtlich: Durch die Vergabepolitik von Mitteln an NGOs kann viel ermöglicht oder eben auch verhindert werden.

Beim Geld angekommen, setzt sich Sabine Dlugosch von der Indienhilfe Herrsching zuletzt mit dem Wunsch vieler Spenderinnen und Spender auseinander, dass kein Euro in die Verwaltung gehen solle, sondern jeder Euro, den die NGOs in der Entwicklungszusammenarbeit ausgeben, direkt der Zielgruppe zu Gute kommen soll. Am Beispiel einer kleinen NGOs zeigt sie auf, welche unverzichtbaren Qualitätsstandards professionelle Hilfe braucht, und was diese kosten muss. Denn auf Transparenz und Wirksamkeit werden Spendende nicht verzichten wollen.

Zuletzt ist es mir ein Anliegen, mich besonders bei Frau Prof. Rotering-Steinberg für die fachliche Begleitung des Erstellungsprozesses dieses Themenheftes zu bedanken.

Den Leserinnen und Lesern wünsche ich aufschlussreiche Leseerfahrungen.

\section{Literatur}

Stickler, A. (2005). Nichtregierungsorganisationen, soziale Bewegungen und Global Governance, Eine kritische Bestandsaufnahme. Bielefeld: transcript verlag. 